\title{
Development of a Technique and Method of Testing Aircraft Models with Turboprop Engine Simulators in a Small-scale Wind Tunnel - Results of Tests
}

\author{
A. V. Petrov, Y. G. Stepanov, M. V. Shmakov
}

This report presents the results of experimental investigations into the interaction between the propellers (PS) and the airframe of a twin-engine, twin-boom light transport aircraft with a П-shaped tail. An analysis was performed of the forces and moments acting on the aircraft with rotating PS. The main features of the methodology for windtunnel testing of an aircraft model with running Ps in TsAGI's T-102 wind tunnel are outlined.

The effect of 6-blade Ps slipstreams on the longitudinal and lateral aerodynamic characteristics as well as the effectiveness of the control surfaces was studied on the aircraft model in cruise and takeoffllanding configurations. The tests were conducted at flow velocities of $V_{\infty}=20$ to $50 \mathrm{~m} / \mathrm{s}$ in the ranges of angles of attack $\alpha=-6$ to $20 \mathrm{deg}$, sideslip angles of $\beta=-16$ to 16 deg and blade loading coefficient of $B=0$ to 2.8. For the aircraft of unusual layout studied, an increase in blowing intensity is shown to result in decreasing longitudinal static stability and significant asymmetry of the directional stability characteristics associated with the interaction between the Ps slipstreams of the same (left-hand) rotation and the empennage.

Keywords: windtunnel testing, propeller slipstream, engine failure, test methodology.

\section{Introduction}

The propeller slipstream and airframe interaction is one of the most important problems in the aerodynamic design of an aircraft, especially in the case of a heavily - loaded Ps [1], [2].

The high efficiency of turboprop engines is a reason for their widespread applications. However, effective thrust of turboprop engines depends on their position on an aircraft. The Ps slipstream has an essential influence on the lift and stability and controllability characteristics of an aircraft due to interaction with the wing, fuselage and empennage.

On the other hand, the nacelles, fuselage, wing and other aircraft components influence the flow velocity distribution over the Ps plane of rotation, and as a consequence alter the aerodynamic loads on the Ps blades and their thrust characteristics in comparison with free-stream flow.

Modern computation methods are mainly based on ideal fluid theory, and are not able to fully reveal and take into account the above-mentioned effects. The use of experimental methods is therefore the main way to study the problems of Ps and airframe interaction. This is especially true in the case of aircraft of unusual layout with an unusual position of the engines and empennage.

This report presents the results of experimental investigations of the model of a twin-engine light transport aircraft of unusual twin-boom layout with a $\Pi$-shaped tail. The main feature of the airframe is the twin-fin immersed in the Ps slipstream. In this case the rudder effectiveness may be increased significantly due to the slipstream flow. However, the lateral stability and controllability characteristics can be significantly influenced by the engine power setting as well as by the angles of attack and by the deflection of the wing high-lift devices. The tests in TsAGI's T-102 subsonic wind tunnel were aimed at studying the peculiarities of these effects on the aerodynamics, stability and controllability of the aircraft model in cruise and takeoff/landing configurations.

\section{Aircraft model}

The general geometry of the light transport aircraft (LTA) model is shown in Fig. 1. LTA is a twin-boom high-wing monoplane with a П-shaped tail.

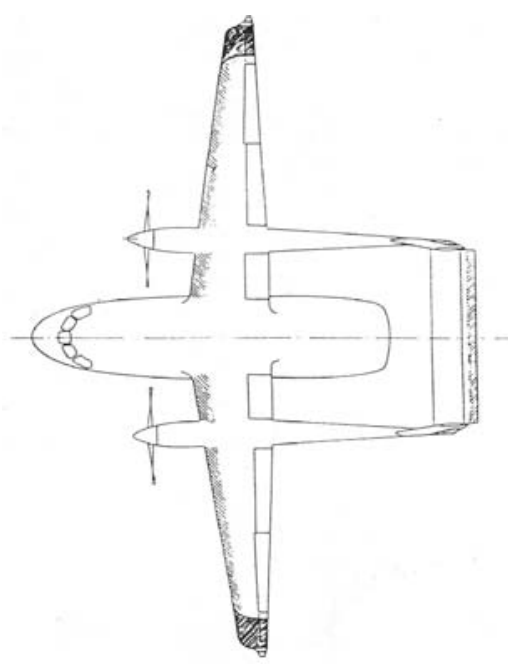

Fig. 1: Model LTA 
The wing aspect ratio is $\lambda=10.9$, wing area $S=0.54 \mathrm{~m}^{2}$, $\operatorname{span} l=2.43 \mathrm{~m}$ and mean aerodynamic $\operatorname{chord} \bar{c}=0.24 \mathrm{~m}$. The double-slotted and double-section flaps can be deflected to angles of $\delta_{\mathrm{f}}=25 \mathrm{deg}$ (takeoff position) and $50 \mathrm{deg}$ (landing position). The twin-fin vertical tail has a relative area of $S_{V T}=0.3$ and a relative arm of $L_{V T}=0.35$. The corresponding relative parameters of the horizontal tail are $S_{H T}=0.25$ and $L_{H T}=3.8$.

The model power plant consists of two 6-blade Ps of left-hand rotation (as viewed forward) with a diameter of $D_{S}=0.36 \mathrm{~m}$, driven by electric drives each with a power of $N=5 \mathrm{~kW}$. The blades are manufactured of a moulded reinforced carbon plastic. They can be assembled on the faired hub (spinner) at the desired blade setting angles in the range $\varphi_{\mathrm{B}}=13.3-29.44 \mathrm{deg}$. The $\mathrm{Ps}$ speed in the range $n=0-65001 / \mathrm{min}$ was measured by an internal photoelectric transducer.

\section{Test methodology}

A technique was developed for testing the model with operating Ps. It consists of two parts:

1) determination of the isolated model power plant thrust;

2) a test methodology for the full aircraft model with two running Ps.

\subsection{Methodology for measuring of Ps thrust}

The methodology is based on measurements of the forces and moments with mechanical balances AB-102 acting on an isolated nacelle with Ps on and off and elimination of the influence of the supporting devices and communications by using calculation and experimental methods.

Determined as a result of balance measurements carried out at flow velocities of $V_{\infty}=0-45 \mathrm{~m} / \mathrm{s}$, Ps speeds $n=0-6500$ $1 /$ min and blade setting angles of $\varphi_{\mathrm{B}}=13.3-29.44 \mathrm{deg}$ were: - available range of thrust $P$ and thrust coefficient

$$
\alpha_{s}=P / \rho_{\infty} n_{s}^{2} D_{s}^{4},
$$

- available range of blade loading coefficient

$$
B=P / 0.25 q_{\infty} \pi D_{s}^{2},
$$

- possible range of Ps advances ratio

$$
\lambda_{s}=V_{\infty} / n_{s} D_{s},
$$

- requirement power $N_{s}$ and power coefficient $\beta_{s}$ range, where

$$
\begin{aligned}
& N_{s}=M_{s} \omega, \\
& \beta_{s}=N_{s} / \rho_{\infty} n_{s}^{3} D_{s}^{5}=2 \pi M_{s} / \rho_{\infty} n_{s}^{2} D_{s}^{5},
\end{aligned}
$$

$V_{\infty}, q_{\infty}, \rho_{\infty}-$ free stream parameters,

$n_{s}$ - propeller speed, $1 / \mathrm{s}$

$\omega$ - propeller angular velocity, $\omega=2 \pi n_{s}$,

$M_{s}$ - torque moment on the Ps shaft.

In Fig. 2, the thrust coefficient $\alpha_{s}$ is plotted as a function of advanced ratio $\lambda_{s}$ at $\varphi_{B}=29.44 \mathrm{deg}$ and $n=50001 / \mathrm{min}$. The relationship $\alpha_{s}\left(\lambda_{s}\right)$ is corrected for the nacelle and the interference of supporting devices, and agrees satisfactorily with the test data of a large-scale model of the similar Ps in TsAGI's T-104 large wind tunnel.

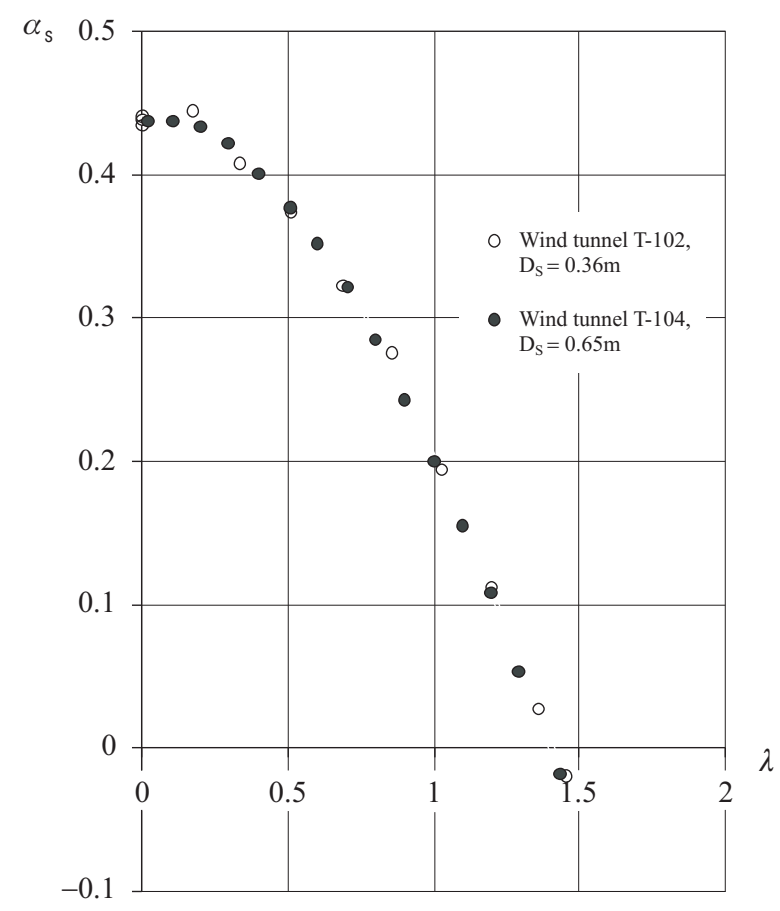

Fig.2 Propeller thrust coefficient $\alpha_{s}$ versus advance ratio $\lambda$ Propeller CB-34-01

\subsection{Test methodology for the aircraft model with running Ps}

The investigations of the aerodynamic characteristics of the aircraft model in cruise and takeoff/landing configurations were conducted in the ranges of angles of attack $\alpha=-6$ to $20 \mathrm{deg}$, sideslip angles of $\beta=-16$ to $16 \mathrm{deg}$ and loading coefficient $B=0-2.8$. The values $B \leq 0.3$ correspond to the cruise flight regime and range $B=1.0-2.8$ corresponds to the takeoff/landing regimes.

The values of $\lambda_{s}$ and the corresponding required values of the propeller speed $n_{s}$ were determined according to the needed values of $B$. The values of $V_{\infty}$ were determined under conditions of maximum possible values of Re number for the model and electrodrive power limitation.

When calculating the aerodynamic coefficients, the forces and moments were referenced to the flow dynamic pressure and base wing area. The pitching moments, in addition, were referenced to the mean aerodynamic chord, and the rolling and yawing moments were referenced to the wing span. The moment values were determined relative to the conditional center of gravity position at a distance of $x_{g}=0.257 \bar{c}$ from the MAC forward edge.

\section{Aircraft model test data analysis}

\subsection{Longitudinal aerodynamic characteristics}

Tests of the LTA with running Ps on and off for cruise $\left(\delta_{f}=0\right.$, landing gear retracted $)$, takeoff $\left(\delta_{f}=25 \mathrm{deg}\right)$ and landing $\left(\delta_{f}=50 \mathrm{deg}\right)$ configurations were conducted in the ranges of angles of attack $\alpha=-6$ to $20 \mathrm{deg}$ and coefficients $B=0-2.8$, including also the autorotation regime (AP). 


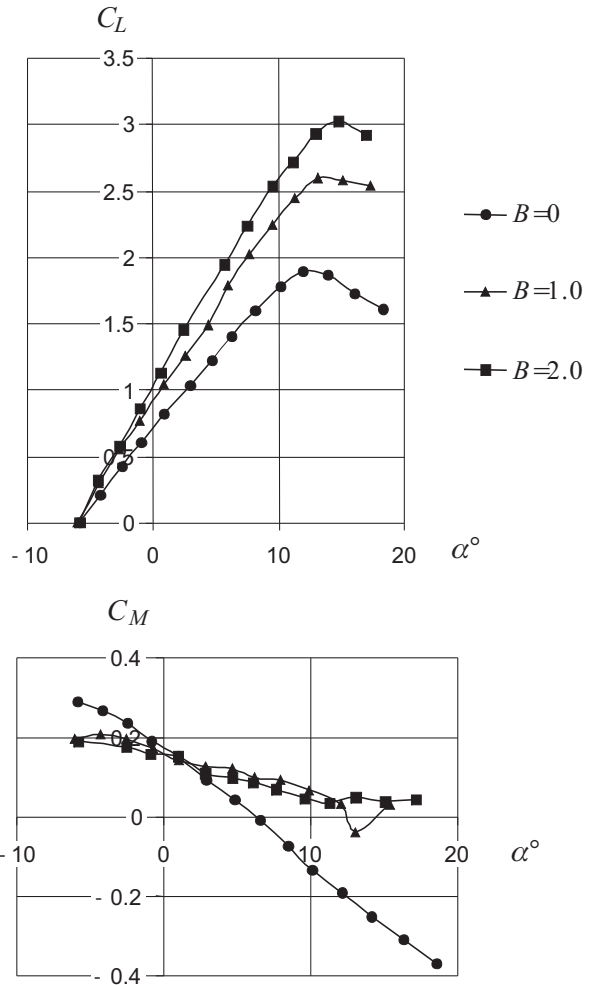

Fig. 3: Longitudinal aerodynamic characteristics

Fig. 3 is an example of the test results for the takeoff configuration. An increase in blow intensity (loading coefficient $B$ ) increases the derivative $C_{L}$, maximum lift coefficient and critical angle of attack, improving effectiveness of the flaps and essentially decreasing the longitudinal stability (see Fig. 3). The aerodynamic center moves from $\bar{X}_{F}=53.7 \% \bar{c}$ (at $B=0$ ) to $\bar{X}_{F}=32 \% \bar{c}$ at $B=2$. This is mainly associated with the action of the transverse force on the Ps and the Ps slipstream effect on the flow about the horizontal tail. The test results show that the aerodynamic center of the model without the horizontal tail shifts to a much lesser degree, and the pitching moment increases in the nose-down direction due to the increased effectiveness of the flaps blown by the Ps slipstreams.

A substantial feature of an aircraft with two left-hand-rotation Ps is the appearance of yawing moments with zero sideslip (see Fig. 3). This is mainly associated with the action of the two slipstreams swirled in the same direction on the flow about the vertical tail immersed in the wake produced by the Ps.

\subsection{Lateral aerodynamic characteristics}

The effect of the running Ps on the lateral stability of the aircraft model is comparatively small for the configurations tested. However, the directional stability characteristics vary considerably with varying blowing intensity (Fig. 4). The variations of directional stability characteristics with sideslipping, rudder deflected, and running engines results mainly from the action of two factors: non-uniform variations of the rudder effectiveness with sideslip angle (due to displacement of the slipstream core in the direction of the Ps rotation), and the peculiarities of the interaction between the two left-hand swirled Ps slipstreams and the vertical tail. As a consequence,
Takeoff configuration

$\delta_{f}=25^{\circ} / 25^{\circ} ; \quad x_{T}=0.25$
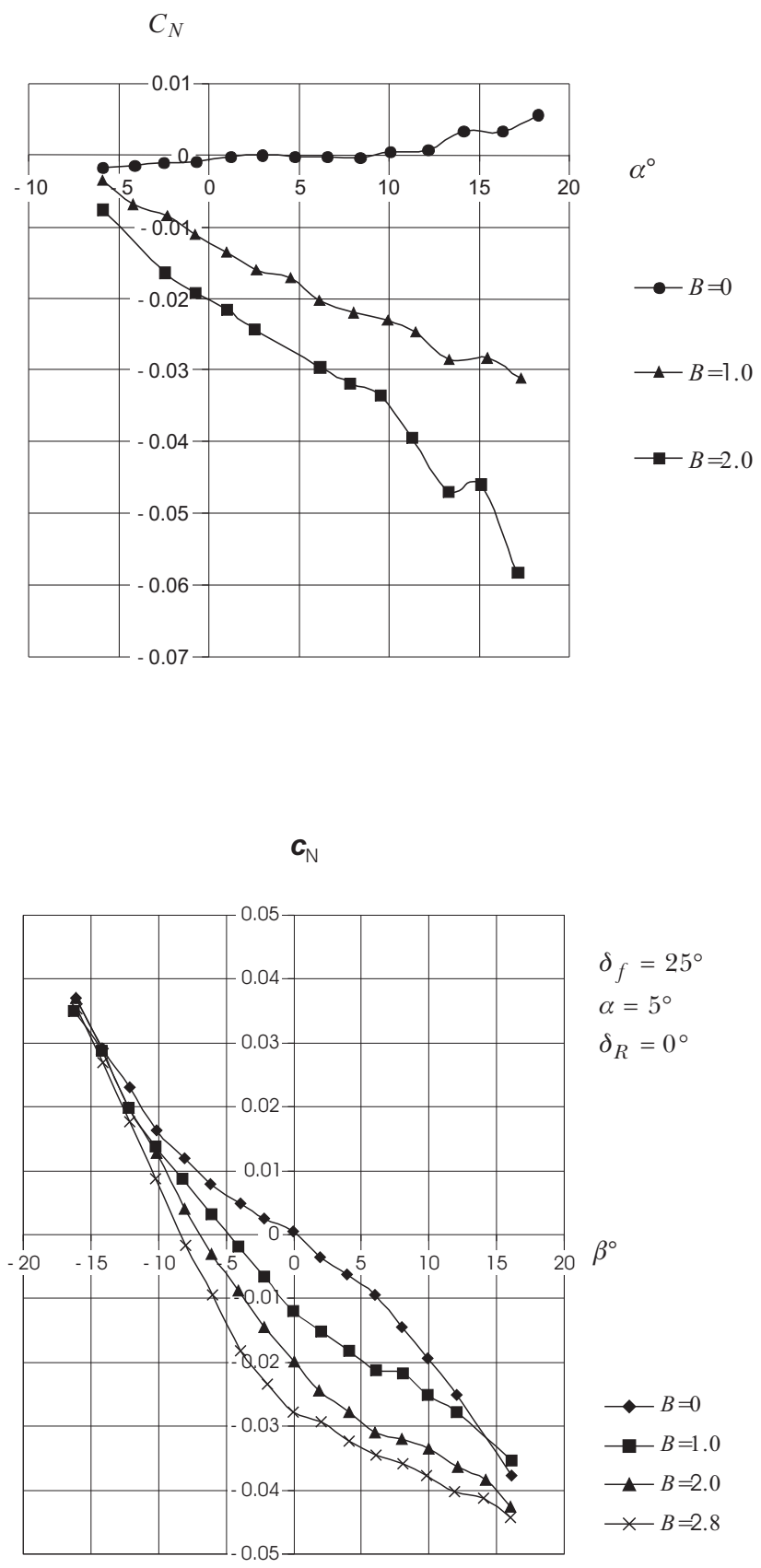

Fig. 4: Lateral aerodynamic characteristics

an increase in the loading coefficient $B$ from 0 to 2.8 leads, in addition to the variations of $C_{N}$ with $\beta=0$, to "nonsymmetrical" variations in directional stability: this increases with the left wing slip $(\beta<0)$ and decreases with the right wing slip ( $\alpha=0-15 \mathrm{deg})$.

\subsection{Effectiveness of control surfaces}

The effectiveness of the rudder with the propellers operative significantly increases with increasing blade loading coef- 
ficient $B$; in this case the region of maximum increments of the yawing moment shifts towards negative angles of sideslip $(\beta=-5$ through $-10 \mathrm{deg})$, i.e. in the direction of rotation of the propellers (Fig. 5). The effectiveness of the rudder on the model in takeoff configuration at an angle of attack of $\alpha=5^{\circ}$ and $B=2$ increases by a factor of $1.6-1.7$ at $\beta=0$, and by a factor of $1.8-2.1$ at $\beta=-6$ through $-10 \mathrm{deg}$.

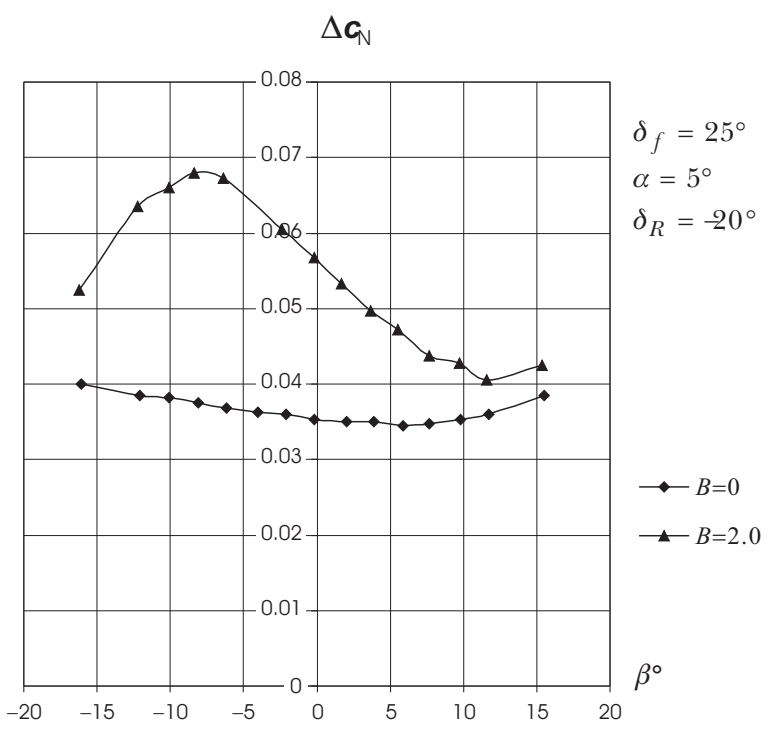

Fig. 5: Rudder effectiveness
The effectiveness of the ailerons and elevator in the operational angle-of-attack range depends rather insignificantly on the power setting.

\section{Effect of engine failure on the aerodynamic characteristics}

Studies into one-engine-inoperative (left- or right-hand) situations were performed on the aircraft model in takeoff configuration ( $\delta_{f}=25 \mathrm{deg}$, landing gear extended) at a propeller area load factor $B=2$, corresponding to the maximum takeoff engine power. The propeller blades of the filed engine are in feathered pitch (FP).

Fig. 6 demonstrates the longitudinal aerodynamic characteristics of the model with both engines operative $\left(B_{r}=2\right.$; $\left.B_{l}=2\right)$, with the right engine failed $\left(B_{r}=\mathrm{FP} ; B_{l}=2\right)$ and with the left engine failed $\left(B_{r}=2 ; B_{l}=\mathrm{FP}\right)$, as well as with both Ps windmilling (WM).

The change-over from WM to $B=2$ results increases the derivative $C_{L}^{\alpha}$, the maximum lift coefficient and critical angle of attack, decreases the degree at static stability, and decreases the degree at the longitudinal (propulsive) thrust component (shift of the drag polar to the negative $C_{D}$ domain).

Failure of the left or right engine results in approximately the same decrease in the increments of the lift and longitudinal force. Besides, with one engine failed the longitudinal static stability of the aircraft model is restored to a significant degree.
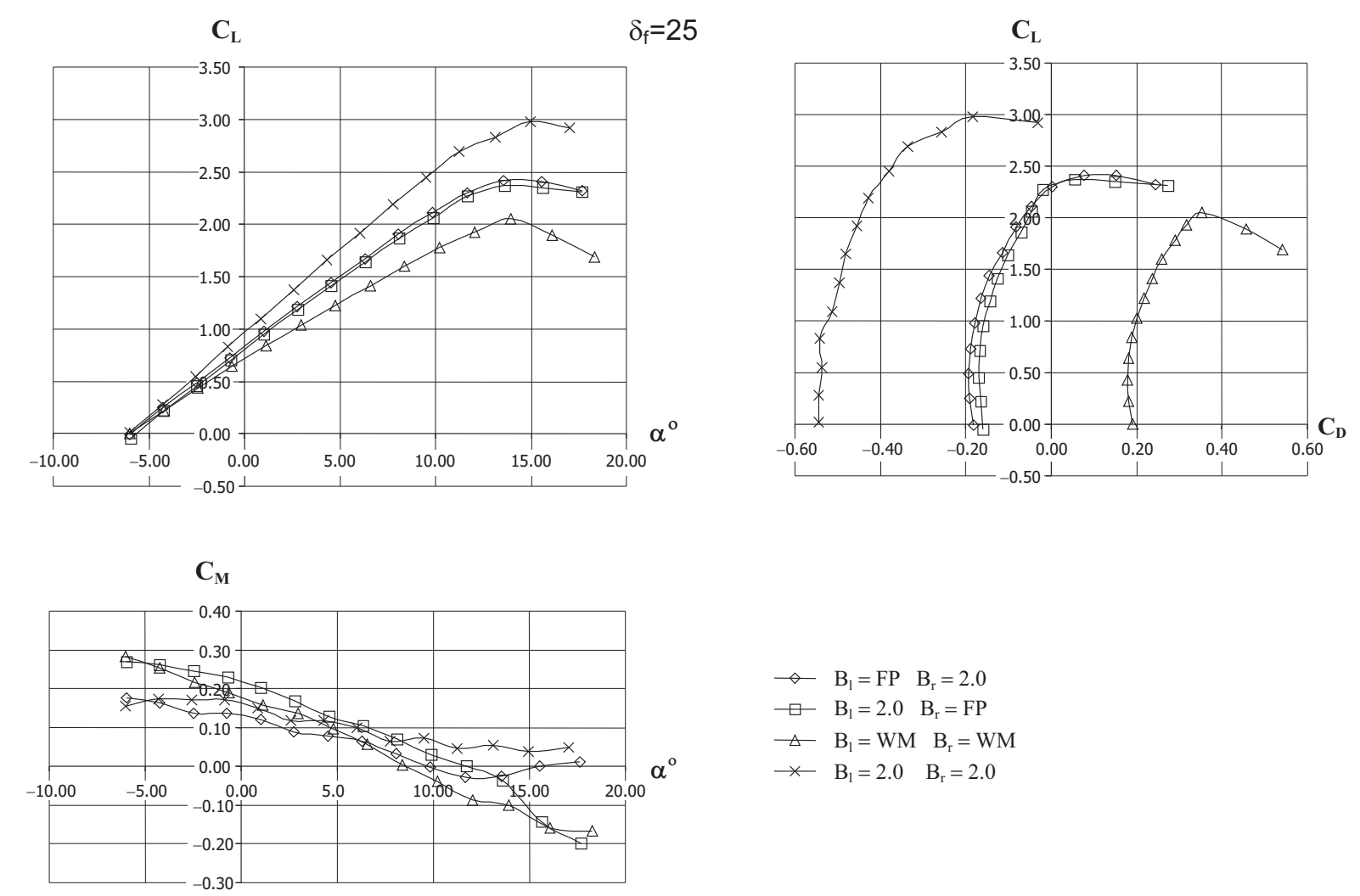

Fig. 6: Longitudinal aerodynamic characteristics of the model 
A special feature of the aircraft layout with the twin-fin tail in propeller slipstreams is the generation of a significant directional moment of negative sign, which increases with the angle of attack and with loading coefficient B (see Fig. 3). This is attributed to the effect of flow angularity on the vertical tail, created by the swirling slipstreams behind the Ps with the same direction of rotation (left-handed).

The influence of the operating Ps on the rolling moment is insignificant up to $\alpha \approx 10^{\circ}$. However, the failure of any engine results in the generation of rolling moments opposite in sign and approximately equal in magnitude at the identical angles of attack.

\section{Conclusion}

Investigations of a model of a twin-engine transport aircraft with an uncommon twin-boom layout and running propellers have shown that an increase in the blowing intensity leads to enhancement of the lifting ability of the aircraft and degradation of its longitudinal static stability. It was found that a twin-fin tail unit in the propellers slipstreams increases the rudder effectiveness by a factor of 1.5-2 with the running propeller in takeoff regime.

However, because of the interaction of the swirling slipstreams behind propellers with the same direction of rotation and the vertical tail, a significant yawing moment appears on an aircraft with the considered layout (even at zero sideslip angle) and its directional stability characteristics vary noticeably.

Failure of the right (critical) engine leads to an additional significant increase in the yaw moment, which poses the problem of ensuring the aircraft's directional trim.

\section{References}

[1] Petrov A. V.: Aerodynamics of STOL Aircraft with Wings Blowing by Turbofan Exhaust Jets and Propfan Slipstreams. AIAA-93-4832, 1993.

[2] Viskov A. N., Kishalov A. N., Petrov A. V.: Problems of designing advanced turboprop aircraft. Proceedings of the International Conference "Aviation Technologies 2000" Zhukovsky, 1997.

Dr. Albert V. Petrov

Dr. Yury G. Stepanov

Dr. Michael V. Shmakov

Aerodynamic Department

Central AeroHydrodynamic Institute (TsAGI)

Zhukovsky str. 1

Zhukovsky, 140180, Russia 